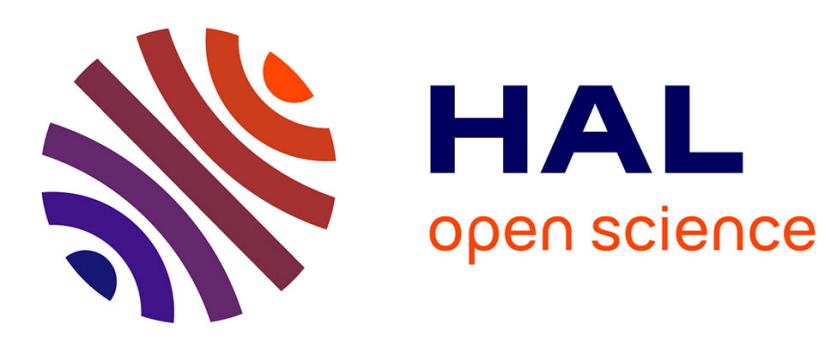

\title{
Un cas de déviance dans les classes populaires : les seuils d'entrée dans les troubles alimentaires
}

\author{
José Luis Moreno Pestaña
}

\section{To cite this version:}

José Luis Moreno Pestaña. Un cas de déviance dans les classes populaires : les seuils d'entrée dans les troubles alimentaires. Cahiers d'Economie et de Sociologie Rurales, 2006, 79, pp.67-95. hal-01201124

\author{
HAL Id: hal-01201124 \\ https://hal.science/hal-01201124
}

Submitted on 17 Sep 2015

HAL is a multi-disciplinary open access archive for the deposit and dissemination of scientific research documents, whether they are published or not. The documents may come from teaching and research institutions in France or abroad, or from public or private research centers.
L'archive ouverte pluridisciplinaire HAL, est destinée au dépôt et à la diffusion de documents scientifiques de niveau recherche, publiés ou non, émanant des établissements d'enseignement et de recherche français ou étrangers, des laboratoires publics ou privés. 


\section{Un cas de déviance dans les classes populaires : les seuils d'entrée dans les troubles alimentaires}

José Luis MORENO PESTAÑA 


\section{José Luis MORENO PESTAÑA*}

\section{A case of deviance in the working classes: On the threshold of eating disorders}

Summary - This text proposes a sociological analysis of eating disorders in the popular classes. It takes into account Ian Hacking's theory of ecological niches of transitory mental illness, giving this theory a sociological formulation. The article proposes some bypotheses about the handicaps and the counter-handicaps of the popular classes in relation to eating disorders. The text, except for being based on empirical investigation, presents a profile of a contemporary somatic culture in the working class, especially among the youth, bighlighting the differences and similarities to the dominating class.

Key-words : sociology of mental illness, sociology of anorexia and bulimia, working classes, body, alimentation, deviance theory

\section{Un cas de déviance dans les classes populaires : les seuils d'entrée dans les troubles alimentaires}

Résumé - Ce texte propose une analyse sociologique des troubles alimentaires dans les classes populaires. Il fait appel à la théorie des niches écologiques des maladies mentales transitoires, telle qu'elle est définie par Ian Hacking, et lui donne une re-formulation sociologique. L'article propose des hypothèses sur les handicaps et contre-handicaps des classes populaires par rapport aux troubles alimentaires. Le texte, résultat d'une recherche empirique, établit un profil de la culture somatique contemporaine des classes populaires, surtout des jeunes, et propose une comparaison avec celle des classes dominantes.

Mots-clés : sociologie, maladie mentale, anorexie et boulimie, classes populaires, corps, alimentation, théories de la déviance

* Departamento de Historia, Geografía y Filosofía, Universidad de Cádiz, Avenida Gómez Ulla, s/n, 11003 Cádiz, Espagne

e-mail : joseluis.moreno@uca.es

Traduction de Philippe Hunt. Je tiens à remercier Muriel Darmon, Didier Fassin, Jean-François Gaspar, Samuel Lézé, Anne Lhuissier, Enrique Martín Criado et Stanislas Morel pour leurs commentaires sur diverses versions de cet article. Des versions antérieures de ce travail ont été présentées lors de séances de discussion au Centre de recherches sur les enjeux contemporains en santé publique et au cours du séminaire « Populations défavorisées et alimentation », organisé par le CORELA à l'INRA d'Ivry-sur-Seine. 


\section{Les déviances et les nosologies}

En avril 2003, Francisca a 14 ans. Elle s'habille en style grunge: t-shirt, pantalon et bottes. Brune, la peau foncée ${ }^{1}$, elle habite un village agricole. Francisca parle avec un accent andalou très prononcé, j’ai du mal à la comprendre.

Francisca s'est rendue, accompagnée de ses parents, dans une association d'aide contre l'anorexie et la boulimie ${ }^{2}$. Un professeur de son lycée lui avait recommandé de faire cette visite. Les parents de Francisca, habillés de manière très soignée, s'expriment avec réserve. Je discerne chez eux la honte que manifestent habituellement beaucoup de ceux qui viennent à l'association; non sans raison, ils pensent qu'il s'agit d'une association qui se consacre au travail avec les «fous ». Par ailleurs, il m'a semblé évident que les parents de Francisca ont la timidité des gens de la «campagne » quand ils arrivent à la capitale. Dans mon journal de terrain, j'ai noté ceci: "travailleurs endimanchés ». Sans aucun doute, l'origine sociale de Francisca et de ses parents est bien plus modeste que celle d'autres usagers de l'association.

Les parents attendent dans le corridor : ils parleront avec une autre psychologue. Francisca entre dans le bureau avec Ana, une psychologue de l'association, et moi.

Francisca raconte qu'elle a commencé à se sentir grosse un an auparavant; pour maigrir, elle a cessé de manger. Cela ne lui a pas posé de problème: ses parents travaillent comme ouvriers agricoles et, de ce fait, elle pouvait éviter le contrôle maternel à midi. Pour le repas du soir, cependant, c'était impossible et elle devait manger normalement. En contrôlant son alimentation, Francisca a réussi à perdre 20 kilos en un an. Aussitôt, Ana, la psychologue, réagit: "C'est beaucoup! " "Pas du tout, répond Francisca, j'étais très grosse!». Elle le constate avec amertume. Plus tard, j'ai noté ceci dans mon journal de terrain : "il était évident pour tout le monde, sauf pour ses parents, que Francisca était très grosse ». Francisca considérait qu'en réduisant son alimentation, elle commençait à manger de manière normale. Avant cela, expliquet-elle, elle mangeait trop: "j'étais capable de dîner d'un sandwich, deux yoghourts et un verre de lait ». Surpris par son amaigrissement et après en avoir parlé avec ses parents, son professeur l'a envoyée à l'association, convaincu que Francisca «avait des problèmes ».

La psychologue fait ensuite dévier la conversation vers la vie familiale de Francisca. Elle dit qu'elle est en conflit avec l'une de ses sœurs et, à présent, avec ses parents aussi. La psychologue lui signale qu'il est normal que ses parents soient

\footnotetext{
${ }^{1}$ Cette pigmentation n'est pas, bien sûr, le résultat d'un bronzage à la plage ou à la montagne, il s'agit en fait de ce que nous appelons souvent ironiquement en espagnol « le hâle du maçon ».

2 Pendant deux ans, j’ai mené un travail de terrain dans cette association. Reçu comme professeur d'université «qui voulait faire des recherches sur l'anorexie et la boulimie », ma relation à cet objet de recherche s'est modifiée au cours du temps : au départ, je n'ai eu accès qu’à des conversations avec les responsables, puis j'ai pu participer aux entretiens que les psychologues réalisaient avec les usagers de l'association.
} 
préoccupés à son sujet. Francisca acquiesce. Elle raconte qu'elle «séchait les cours » et qu'un jour, en compagnie de son petit ami, elle est restée dans la rue jusqu'à trois heures du matin. Ana l'interroge sur ses problèmes intimes. Francisca répond: « je dois maigrir, mais je n'arrête pas de manger des sucreries». Ana lui donne ce conseil : "c'est une question d'habitude : oublie les sucreries et mange des fruits, des légumes!». Francisca répond: «ce n’est pas facile! Les légumes, c'est pour les lapins! ». Nous éclatons de rire tous les trois. Puis, la psychologue, nettement plus à l'aise, cherche à se concentrer sur la vie sentimentale de Francisca, mais celle-ci n'a pas envie d'en parler. À la fin de l'entrevue, Ana demande à Francisca si elle sait ce qu'est l'anorexie. Elle dit que non. Ana insiste: «que penses-tu de l'anorexie?». «Bon, répond Francisca, c'est une affaire de fous, non?». À la fin de l'entrevue, Francisca annonce qu'elle a l'intention de quitter le lycée et de commencer des études d'esthéticienne.

Après cette conversation, j'assiste à une réunion de synthèse entre Ana et la psychologue qui a rencontré les parents. Selon ses parents, Francisca est «obsédée » par son physique et son professeur au lycée a mis cette obsession en rapport avec une «maladie » éventuelle de leur fille. En même temps, Francisca passe, selon ses parents, par une étape très « rebelle », avant tout parce qu'elle fréquente pas mal de garçons. Ana et l'autre psychologue discutent. J'ai noté ceci dans mon journal de terrain: "la position d'Ana est que Francisca n'a pas le moindre problème. Elle a besoin d'aide pour étudier, c'est tout; l'autre psychologue considère qu'elle peut avoir des problèmes (alimentaires). Francisca, très contente, est partie et a pris rendez-vous pour le lendemain. Son diagnostic est encore indéfini $»$.

Je passe à un autre cas. Pendant une entrevue collective avec un groupe d'étudiants, Sara m'explique qu'elle a souffert d'anorexie. Elle a 20 ans, mesure 1 mètre 80 , est fort mince; elle s'habille de manière très élégante et $a$, auprès des professeurs de la faculté, la réputation d'être une étudiante «brillante ». Les parents de Sara sont de petits agriculteurs qui, avec le temps, ont ouvert une épicerie. Avant de quitter la maison pour étudier, Sara habitait avec eux dans un petit village de montagnes (5 000 habitants), en Andalousie.

Profitant du fait que ses parents étaient en vacances, Sara a commencé à réduire son alimentation. En même temps, elle a continué à faire du sport et de la danse folklorique. À l'investissement corporel considérable déjà présent dans sa vie, s'est donc ajouté un contrôle draconien de son alimentation.

Je me rappelle très bien. J'avais maigri et, au mois de juillet, je me suis acheté une robe. En août, la robe était beaucoup trop grande. Ma mère m'a regardée comme elle ne l'avait jamais fait et elle m'a giflée. "Qu'est-ce qui t'arrive? ", m'a-t-elle dit, "qu'est-ce que tu vas me combiner? ". C'est la seule fois de ma vie que j'ai rę̧u une gifle de ma mère. Je me suis senti super mal. Je lui ai dit: "Maman, c'est fini..., je sais que ce n'est pas normal ».

Il n'a pas été pas facile pour Sara de recommencer à manger normalement. Sa mère surveillait son alimentation et transforma la maison en une espèce d' « institution totale ». «Elle me faisait des chantages terribles », conclut Sara.

Avant 1993, Sara n'avait jamais entendu le mot "anorexie » et sa mère, pas davantage. Elle n'en est pas sûre, mais elle croit que pendant cette période 
d'autocontrôle corporel intensif, elle n'a pas eu ses règles. Aucun professionnel n'a diagnostiqué une anorexie, aucun proche n'a mentionné la possibilité que Sara soit malade ou présente les symptômes d'une maladie quelconque. Ce n'est que deux ans plus tard, lorsque l'une de ses amies a souffert de troubles alimentaires que Sara «a pigé » qu'elle avait « eu » une anorexie.

Ces histoires ont beaucoup de points communs, et en même temps, on pourrait dire qu'un monde les sépare. Deux jeunes filles, issues de classes populaires, venant toutes deux de foyers économiquement faibles et de milieu rural, ont commencé un processus d'autocontrôle de l'alimentation. Pour ce faire, elles n'ont pas eu recours à un régime prescrit par un professionnel: elles ont simplement cessé de manger. Toutes deux ont été promptement repérées par leurs proches et ont subi une intense mobilisation familiale visant à corriger leur comportement. Les modèles corporels recherchés par Francisca et Sara ne passèrent pas inaperçus et la réaction des familles a été négative. Malgré cela, Francisca et Sara désiraient être «minces» et les restrictions alimentaires secrètes, ainsi que le sport dans le cas de Sara, étaient les voies dont elles disposaient.

Il est facile d'imaginer d'autres réactions possibles et ce dans trois cadres différents. Sara et Francisca auraient pu recourir à un nutritionniste ou à un régime alimentaire pour maigrir, avec l'appui de la famille. Ou alors, Sara et Francisca auraient pu maigrir sans que personne ne s'en scandalise : la morphologie corporelle résultant de leur autocontrôle aurait recueilli les éloges de leur entourage familial ${ }^{3}$. Ou encore, tout en leur reprochant les méthodes brutales qu'elles utilisaient pour maigrir, leurs familles auraient pu leur proposer d'autres méthodes sans pour autant condamner leur désir de maigrir.

C'est une culture corporelle spécifique qui le leur a interdit. Une culture dans laquelle: i) la pratique d'un régime alimentaire ne suscite aucune approbation; ii) les modèles féminins minces déchaînent facilement les inquiétudes du groupe familial ; et enfin iii), l'objectif de maigrir, même chez des jeunes filles, n'est pas considéré comme légitime. Je considère que cette culture corporelle est une culture de classe ou, si l'on préfere, plus précisément une culture corporelle surtout présente dans certaines fractions des classes populaires.

La différence entre Francisca et Sara est très visible. La famille de cette dernière, contrairement à la première, ne disposait pas de la catégorie «anorexie " pour caractériser sa déviance. Francisca entrait dans la première phase d'un circuit professionnel, alors que Sara subissait une expérience de contrôle massif de sa vie, semblable à ce que connaissent beaucoup de personnes caractérisées comme anorexiques, même si aucun diagnostic n'a été fait.

${ }^{3}$ Le cas le plus extrême que je connaisse est celui d'une femme professeur de 30 ans, fille d'un psychiatre et appartenant à un milieu social bourgeois, qui m'a expliqué que malgré son état émacié, obtenu par des méthodes qui combinaient restriction alimentaire et vomissement, elle n'a jamais reçu que des compliments de son entourage. 


\section{Pour une sociologie de la niche écologique de l'anorexie et de la boulimie}

Pour analyser les troubles alimentaires, j’utiliserai le concept, élaboré par Ian Hacking, de niche écologique d'une maladie mentale transitoire ${ }^{4}$. Selon Hacking, une telle niche écologique se compose de quatre vecteurs. Premièrement, l'existence d'une taxinomie médicale capable de définir certains types de conduites à l'intérieur d'un cadre nosologique. Deuxièmement, une polarité culturelle autour d'un certain type de comportement qui serait à la fois objet d'une admiration collective et indice d'un comportement vieux et dégradé. Troisièmement, un vecteur d'«observabilité » qui permette de découvrir le comportement «pathologique ». Et quatrièmement, ce comportement pathologique doit permettre d'atteindre des objectifs vitaux qui seraient inaccessibles d'une manière normalisée. Ce dernier vecteur aide à préciser le recrutement social des individus qui s'embarquent dans des maladies mentales transitoires (Hacking, 2002, pp. 180-182).

Mon utilisation du modèle de Hacking se fonde sur deux présupposés. D'abord, ce modèle ouvre de précieuses possibilités de description sociologique de maladies mentales ${ }^{5}$ telles que l'anorexie et la boulimie. Ensuite, ces possibilités de description élargissent celles qui sont permises par d'autres modèles, sans pour autant les exclure. Par exemple, le modèle de «carrière morale ", élaboré par Goffman (1968,

\footnotetext{
${ }^{4}$ Le concept de maladie mentale transitoire, selon Hacking (2002, p. 34) sert à expliquer comment «certains types de maladie mentale et certains arrangements de symptômes sont fortement présents dans certains lieux et à certaines époques, et absents dans d'autres, même dans le cadre d'une culture à peu près continue. Ce sont là les troubles que j'appelle transitoires, non pas au sens d'apparitions et rémissions au cours de la vie d'une même personne, mais en ce sens qu'ils n'existent qu'à une époque et dans un lieu donnés ». Je tiens à mentionner deux questions, même si elles dépassent l'objet de mon article, car l'une participe de mes présupposés ontologiques de base et l'autre constitue un thème de recherche possible intéressant. La question qui relève de mes présupposés ontologiques est la différence, proposée par Hacking, entre espèces naturelles (ou indifférentes), espèces interactives et espèces hybrides. Les espèces naturelles ne sont pas affectées par les pratiques culturelles (c'est le cas des protons), tandis que les espèces interactives le sont (par exemple, la notion de "femme maltraitée »). Les espèces hybrides mélangent les propriétés des deux autres : c'est le cas de la schizophrénie. Les troubles alimentaires constituent, selon moi, une espèce interactive ou tout au moins hybride. Le thème de recherche possible, à partir de processus d'analyse comparative, serait de savoir si une niche écologique d'une maladie mentale transitoire peut survivre à la disparition des vecteurs qui la composent. Voir Hacking, 2001 (pp. 169-205) et Faucher, 1999 (pp. 16-18 et p. 28).

5 Il me semble intéressant de signaler une question qui accompagne un sociologue lorsqu'il réalise un travail sur l'anorexie et la boulimie. Certains sociologues et anthropologues (peu nombreux, mais agressifs), pour ne pas parler de certains philosophes postmodernes, se scandalisent quand $j$ 'affirme que l'anorexie et la boulimie sont des maladies mentales. Je vais avoir recours à une définition minimaliste de la maladie mentale, proposée par Castel (1980, pp. 192 et 252), qui regroupe bien les choses que je vise lorsque je dis que quelqu'un souffre de maladie mentale : cette personne se trouve dans une impasse existentielle tellement profonde et dans une situation si fragile qu'elle a un besoin urgent qu'on s'occupe d'elle et requiert donc une tutelle, médicale et pédagogiquement fondée. Pour une critique de la tendance impérialiste de trois modèles sociologiques d'explication de la maladie mentale et de leur prétention malencontreuse à monopoliser les principes explicatifs de l'étiologie de la maladie mentale, voir Sánchez Moreno (2002, pp. 35-58).
} 
pp. 180-181), laissait en dehors de son dispositif analytique les personnes qui présentaient des comportements semblables à ceux des malades, mais qui i) n'avaient pas été diagnostiquées, ii) recevaient un traitement à l'extérieur d'un cadre hospitalier, et iii) suivaient une psychothérapie alternative à la psychiatrie. Vu que ma perspective de recherche vise l'étude sociologique des déviances corporelles et alimentaires (celles qui constituent un cas possible de troubles alimentaires, tels qu'ils sont qualifiés et traités par la médecine), le modèle de Hacking m'aide à intégrer analytiquement des cas de figures, aussi pertinents sociologiquement pour l'étude de l'anorexie et de la boulimie selon moi, que ceux de Sara et de Francisca. Cela ne signifie pas que ce modèle m'empêche de construire des itinéraires d'internement diversifiés. Somme toute, la relecture sociologique du modèle de Hacking permet de poser des questions plus larges que celles autorisées par d'autres dispositifs d'analyse sociologique, sans exclure les problématiques posées par ces autres dispositifs ${ }^{6}$.

Parmi les questions sociologiques que le modèle de Hacking permet de poser, je retiendrai les suivantes, sans prétention d'épuiser le sujet : chacune des questions est liée à l'un des vecteurs définis par Hacking. La première question est liée au vecteur de la taxinomie médicale: y a-t-il des différences entre les classes sociales dans l'utilisation des catégories médicales pour caractériser les surenchères corporelles? Mes analyses montrent qu'elles existent encore, mais qu'elles ont tendance à diminuer. La deuxième question dérive du vecteur de la polarité culturelle: les comportements corporels susceptibles d'être caractérisés comme "anorexiques » produisent-ils un jugement ambivalent dans tous les groupes sociaux? Je répondrai que ce n'est vrai que pour certains groupes. Les comportements qui peuvent être lus comme «symptômes» de troubles alimentaires sont associés, dans les fractions des classes populaires que j'ai étudiées, à une recherche délibérée de la beauté qui mérite bien plus de reproches que d'admiration. La troisième question procède du vecteur de l'observabilité : est-ce avec la même rapidité dans tous les groupes sociaux que l'on appréhende comme "pathologique » un comportement alimentaire restrictif? Vu le

${ }^{6} \mathrm{Il}$ m'est arrivé d'entendre un reproche assez surprenant, si l'on se rappelle que la théorie de Hacking est construite pour une part importante à partir de cas cliniques, selon lequel mes descriptions sont microsociologiques, alors que le modèle de Hacking est pensé pour des cadres «macro ». Il va de soi que j'utilise Hacking pour organiser mon matériau et que je ne prétends ni vérifier, ni falsifier sa théorie, chose qui n'aurait pas de sens dans le cadre des sciences sociales. Cependant, comme je l'ai signalé, je considère que cette théorie permet de poser des questions que d'autres théories n'autorisent pas et peut, en même temps, recueillir les apports de ces autres théories. Sur la relation entre théories philosophiques et travail sociologique, je ne résiste pas au plaisir de citer ces phrases de Jean-Claude Passeron: "Il existe, je le pratique et le conseille à mes étudiants, un tout autre usage des philosophes, usage utilitaire, corporatif et intéressé, qui conduit le sociologue malin à fenilleter d'autres pages pour les arracher à leur contexte philosophique. Ce n'est évidemment pas pour s'y dévergonder, mais pour y puiser les concepts et les schèmes capables de nourrir l'observation sociologique par des hypothèses qui n'auraient pas poussé toutes seules sur le terrain aride de l'enquête laissé à ses seules semences. Toute philosophie contient aussi (...) une phénoménologie descriptive : c'est d'ailleurs ce qui fait que les non-philosophes lisent les pbilosophes. De toutes ces phénoménologies, on peut tirer des "idées » (des langages conceptuels) qui permettent d'interroger les phénomènes en revenant aux méthodes sociologiques de l'observation bistorique et de l'enquête sociologique, enrichies d'un beau butin théorique ainsi devenu théorie empirique » (Moulin et Veyne, 1996, p. 290). 
mode d'appropriation corporelle des comportements de régime (on verra qu'il s'agit de régimes basés sur des restrictions corporelles rapides et brutales) dominant dans certaines fractions des classes populaires, ces comportements y sont observés très vite : observabilité et culture somatique ${ }^{7}$ des classes populaires sont, aussi curieux que cela puisse paraître, intimement unies. Enfin, sur la question qui correspond au quatrième vecteur, je soutiendrai deux conclusions. Premièrement, face à certaines analyses, je nierai qu'il existe des conditions sociales de possibilité pour l'anorexie qui seraient liées à l'appartenance aux classes moyennes et hautes. Cependant, deuxième conclusion, je considère qu'une analyse plus fine des modes de reproduction différentielle des classes populaires pourrait montrer comment les surenchères corporelles se convertissent en quelque chose de relativement rare dans leurs milieux de vie; plus que de conditions de possibilité, je me focaliserai sur la description d'une figure possible des conditions d'impossibilité de l'anorexie et de la boulimie.

\section{L'utilisation de catégories médicales pour classer les comportements déviants}

Ce que Hacking analyse lorsqu'il présente ce vecteur, c'est le processus, à l'intérieur de l'univers scientifique et professionnel, d'instauration et d'extension d'une taxinomie médicale capable de définir un type de comportement comme pathologique. Cette question est fondamentale et, en ce sens, il serait essentiel d'étudier l'extension de l'étiquette "trouble du comportement alimentaire " parmi les psychiatres, psychologues et médecins généralistes espagnols, mais ce n'est pas la seule question sociologique que l'on puisse dériver du premier vecteur proposé par Hacking. Je me suis demandé jusqu'à quel point les groupes sociaux sont prédisposés à utiliser les catégories psychiatriques pour gérer des problèmes de déviance corporelle.

Le cas de Sara fournit d'emblée une première réponse. Si personne n'a utilisé la catégorie d'anorexie pour désigner son comportement, c'est parce que cette étiquette leur était inconnue. En effet, les catégories médicales pénètrent inégalement les différents points de la géographie sociale. Les innovations médicales se transforment en savoir profane, fondamentalement, dans les groupes les plus proches socialement du monde médical. Les classes populaires, étant les plus éloignées de ce monde, accèdent avec retard aux innovations, psychiatriques en l'occurrence ${ }^{8}$.

Néanmoins, la configuration familiale de Sara nous invite à ne pas nous contenter de cette explication et à comprendre que tout, dans les classes populaires, ne répond pas au profil sociologique popularisé par une certaine littérature - et celle-ci, comme on le verra dans la dernière section de cet article, ne s'en souvient pas toujours. Le père de Sara, paysan, fils de paysan, converti en petit commerçant, s'est embarqué dans un

${ }^{7}$ La culture somatique d'un groupe social peut être saisie, par exemple, au travers des pratiques d'alimentation, de santé, de sport, d'investissement en vêtements et des schémas de différenciation sexuelle (Boltanski, 1971, pp. 206-209).

8 Comme le signalait Boltanski (1974, p. 68), les normes médicales des classes populaires ressemblent à un manuel d'histoire de la médecine. 
régime alimentaire sans contrôle médical. C'est, selon Sara, un homme "très coquet " et qui « surveille beaucoup son aspect : il déteste avoir du ventre ». La situation est devenue grave et le père a arrêté son régime, sous la pression médicale : "Il commençait à avoir des vertiges, il a été faire des analyses et le médecin l'a obligé à manger ». Sara conteste que sa propre surenchère corporelle ait quoi que ce soit à voir avec son père; de temps en temps, elle dit en riant que le problème de son père est " qu'il est très poseur ».

Il y a ici deux idées importantes qui, bien qu'évidentes, peuvent passer inaperçues. Première idée : vouloir garder la ligne - et a fortiori s'il s'agit d'un père ! est une coquetterie et, de fait, un trait de frivolité qu'il faut corriger dès lors qu'il produit des tracas, des sacrifices ou des problèmes de santé. La possibilité de corriger ce travers avec des méthodes purement profanes, comme le voudrait la mère de Sara, semble aller de soi.

Deuxième idée : sachant que la maladie constitue une certaine situation de prestige, en ce qu'elle libère l'individu de certaines contraintes sociales, une maladie produite par la coquetterie implique un triple privilège. Le privilège d'être malade, ensuite celui de tomber malade par une action volontaire et, enfin, celui de tomber malade pour une question frivole comme la recherche de l'apparence physique. En ce sens, la maladie ne se conformerait pas au modèle d'une " déviance non responsable » que Parsons (1999, p. 411) considérait comme nodal dans la « définition institutionnelle du rôle de malade » : plus que de maladie, il faudrait parler de risque choisi.

Une recherche sur l'alimentation dans les classes populaires, réalisée avec des groupes de discussion ${ }^{9}$, permet de comprendre comment ce schéma fonctionne dans certaines fractions des classes populaires en Andalousie, bien qu'il commence à y coexister avec d'autres. Un groupe de discussion auquel participèrent de petits agriculteurs d'un village andalou 10 montrait bien comment les exceptions alimentaires étaient liées à des concessions domestiques, qui ne deviennent compréhensibles que lorsque quelqu'un est malade :

M. : (Dans ma maison), nous étions six et on mettait de la nourriture pour cinq. On ne mettait jamais de la nourriture pour l'un d'entre nous... C'est-à-dire qu'il ne mangeait pas... C'était mon frère Agustín qui, comme il souffrait d'anémie,... Dans ma maison, nous

${ }^{9}$ La recherche avec des groupes de discussion, réalisée avec Enrique Martín Criado, permet de produire des matériaux empiriques sur 1) les cadres interprétatifs communs à différentes fractions des classes populaires et 2) les différences internes des classes populaires. Les groupes de discussion s'ouvraient sur la question : «avons-nous réussi à avoir une alimentation saine? ». Les groupes devaient enchaîner leur discours sans intervention du modérateur ou avec le moins d'intervention possible, l'objectif étant de vérifier comment se négociaient spontanément dans le groupe les cadres de l'interprétation et quels thèmes y provoquaient débat. Les groupes devaient se composer de personnes socialement homogènes et qui ne se connaissaient pas entre elles, ce qui est très difficile en milieu rural. En tout, nous avons mis sur pied 11 groupes de discussion, en tenant compte des divisions de sexe, de classe d'âge, de contextes urbain et rural, ainsi que les divisions entre femmes adultes. Les premiers résultats de cette recherche sont repris dans Martín Criado et Moreno Pestaña (2005). Lorsque je me réfererai à un groupe de discussion, je signalerai en note ses caractéristiques les plus pertinentes.

10 Groupe d'agriculteurs âgés de 43 à 73 ans (majorité de gens de plus de 70 ans), dont la plupart n'ont pas fait d'études, sauf deux d'entre eux qui ont fait des études primaires. 
mangions tous, nous étions six et nous étions cinq à manger et on mettait de la nourriture pour cinq, pour un (on mettait une nourriture spéciale).

C'est que, s'il ne mangeait pas ça, il ne mangeait pas, bien sûr, et mon frère, comme il ne mangeait pas, tu devais lui mettre autre chose parce que s'il ne mangeait pas, il se mourait.

F. : Bon, mais ça, c'est... un problème de maladie... c'est un problème de maladie.

M. : Quand il a fait son service militaire, il a attrapé une anémie et il allait mourir. Il était babitué et, comme il ne mangeait pas..., il n'avait pas faim non plus. Je te dis, moi, qu'avant ils nous mettaient ce qu'ils avaient. Avant, j'ai plus ou moins un mètre soixante..., moi, au service, j'étais un des plus grands et, maintenant, mes fils ont tous un empan de plus que moi, parce que l'alimentation s'est améliorée...

$R$. : Parce qu'ils ne travaillent pas, en plus. Dans ma maison, je mangeais une banane avec plein de saletés quand j'avais six ans.

M. : Combien de bananes et de pommes as-tu mangé que tu n'avais pas cueillies dans ton jardin? Avant, celui qui mangeait des bananes, c'est qu'il était malade.

Certes, comme le signale le dialogue du groupe de discussion, les contraintes matérielles ont diminué et la discipline entourant l'alimentation domestique est moindre. Quoi qu'il en soit, l'expérience de ce groupe est tout ce qu'il y a de plus éloignée de la promotion de l'autocontrôle corporel. En premier lieu, leur expérience quotidienne impose un contrôle domestique très fort de l'alimentation : un régime alimentaire spécial n'est concevable que quand on tombe malade - maladie qui, comme l'explique l'intervention à diverses reprises, procédait d'un agent extérieur et mettait l'individu fortement en danger : « il a fait son service militaire, il a attrapé une anémie et il allait mourir ». En second lieu, les processus de réflexivité corporelle liés à des objectifs esthétiques n'ont pas cours dans leur existence. Le problème des régimes de beauté, introduit par le modérateur et qui n'a donc pas surgi spontanément dans le groupe, était considéré comme un "piège »: les gens ont une constitution spécifique - il y a des gros, de même qu'il y a des blonds - et tous les régimes alimentaires imaginables sont incapables de modifier la morphologie d'une personne corpulente :

F. : Vous avez déjà vu à la télévision un mannequin qui pèse 120 kilos ? (...) Arrive une fille mince comme un doigt qui mesure un mètre soixante-dix de haut (...). Pourquoi vous ne mettez pas ou ils ne mettent pas une femme comme la Pepa de Simón? Une de celleslà et que je la voie devenir mince comme un doigt. Comme si j'allais croire ça. Mais si tu en amènes une comme un jonc, elle sera toujours comme un jonc.
A. : (veut raconter une blague) Ici à Jaen, il y avait un bomme qui était gros...
F. : (coupant A.) Et il devait bien mourir gros... s'il était gros...

La seule manière de maigrir, vu que les régimes gradués sont une "arnaque », consiste à cesser de manger. Or, la nature des gros les oblige à manger beaucoup. Donc, un régime alimentaire ne peut être qu'inefficace. Le corps comme espace possible d'un projet de construction personnelle est absolument absent du discours de ce groupe, dans lequel se concentrent des gens qui ont plus ou moins le même âge et la même condition sociale que les parents de Sara ${ }^{11}$.

${ }^{11}$ Ceci permet de calibrer le degré de déviance que supposait le comportement du père de Sara. 
Bien sûr, d'autres groupes de discussion, situés dans des contextes plus urbains et regroupant des personnes plus jeunes, produisirent des discours différents. Dans tous ces groupes, le problème de l'anorexie constitue un thème de réflexion, alors qu'il n'apparaissait pas une seule fois dans le groupe de discussion composé d'agriculteurs plus âgés. Ainsi, dans un groupe de discussion composé de travailleuses qui suivaient un régime alimentaire ${ }^{12}$, une femme expliqua que sa fille toute jeune - au moment où le groupe se réunissait elle avait 11 ans - avait fait l'objet d'un diagnostic d'anorexie à deux ans ("Je croyais que le médecin était fou », dit-elle); le groupe était d'accord avec l'idée qu'il s'agissait d'un problème grave dont ils rendaient responsables les médias et les tailles impossibles popularisées par les mannequins. Cependant, il y a toujours deux registres concernant l'attitude familiale recommandable: d'une part, vu que l'anorexie est un «caprice » (une « déviance responsable »), des méthodes profanes peuvent être utilisées (la mère de la fille à problèmes annonce qu'elle va l'obliger à manger «à coups de gifles »); d'autre part, la volonté de maigrir est considérée comme «normale » et sa dérive anorexique possible constitue une véritable maladie (une « déviance non responsable»). Les parents de Francisca présentent la même ambivalence: leur fille est rebelle aux préceptes de ses parents, mais il se peut qu'elle soit aussi malade, comme le leur suggère son professeur de lycée, hypothèse qu'ils prennent au sérieux en consultant une association spécialisée dans les troubles alimentaires.

C'est le premier registre qui est à l'œuvre dans le cas de Sara. Il n'a pas disparu, mais il coexiste avec la conscience contradictoire que l'anorexie est un trouble résultant à la fois de la frivolité et de quelque chose qui échappe au contrôle des sujets - une maladie. Des positions semblables ont été établies dans un groupe de jeunes étudiants ${ }^{13}$ et dans un groupe de travailleurs entre 30 et 50 ans ${ }^{14}$. Les catégories médicales sont de plus en plus présentes, mais elles n'annulent pas pour autant des schémas d'appréhension du monde qui stigmatisent la volonté de maigrir en l'identifiant avec l'oisiveté, le manque de maturité et l'irresponsabilité, et légitiment de ce fait les méthodes profanes de gestion de la déviance.

L'existence d'un tel double registre profile une sorte de conscience ambiguë. Personne ne sait comment cette ambiguïté se résoudra, si elle persistera ou si elle cessera, et si elle penchera en faveur d'un des deux pôles de tension qui la constituent. Quoi qu'il en soit, il est difficile de concevoir que, si elle penche vers l'un des pôles de l'ambiguïté en éliminant l'autre, la priorité positive retombe sur le pôle qui considère les troubles alimentaires comme une frivolité. La stigmatisation de

12 Un groupe de femmes qui suivaient ou avaient suivi un régime de réduction de poids pendant une période continue d'au moins un mois et dont les âges s'étalaient de 39 à 50 ans. La majorité d'entre elles était maittresse de maison (il y avait deux travailleuses : une employée domestique et une vendeuse de produits cosmétiques).

13 Des étudiants d'université qui, n'étant pas originaires de Grenade, habitaient à ce momentlà des appartements pour étudiants. Leurs parents sont ouvriers ou employés et ont fait des études non universitaires.

${ }^{14}$ Des travailleurs manuels salariés, dont l'un devenu petit propriétaire d'un commerce familial, mariés avec des enfants, certains ayant fait des études secondaires. 
la grosseur, avec la tension esthétique qu'elle impose, la connaissance en matière de troubles alimentaires et la propension des médecins à les diagnostiquer sont de plus en plus présentes dans les secteurs populaires. La taxinomie médicale peut ainsi devenir graduellement une ressource quotidienne avec laquelle on déchiffre les surenchères corporelles déviantes.

Les vecteurs d'une niche écologique d'une maladie mentale transitoire peuvent être conçus comme étant chacun un seuil d'entrée dans la maladie. Quand ils sont franchis, la maladie est installée et les possibilités alternatives de dénomination et de gestion du comportement sont abolies. Le seuil configuré par les taxinomies médicales imprègne fortement, bien qu'encore de manière précaire, la conscience des secteurs populaires que j'ai étudiés : il devient plus facile pour une personne de les utiliser en vue de caractériser le contrôle rigide de son alimentation, et pour son entourage d'en faire autant. Le seuil d'entrée dans les troubles alimentaires représenté par la taxinomie médicale devient aussi de plus en plus facile à franchir pour ceux qui vivent dans les lieux les plus dépossédés, les plus dominés de l'espace social.

Jusqu'ici, j'ai parlé de la manière dont se codifient les surenchères corporelles dans les classes populaires et la place qu'y prend la connaissance provenant du monde des experts au sujet des troubles alimentaires. Il faut aussi se poser la question inverse: comment se codifient, depuis le monde des experts, les surenchères corporelles des gens issus des classes populaires? De fait, la réponse à cette question n'est pas évidente. Je considère qu'il faut la poser comme une des questions que le deuxième des vecteurs de la niche écologique impose à la description sociologique. En fin de compte, les signifiés associés au trouble par le monde des experts offrent aux individus des possibilités de se forger une identité.

\section{La polarité culturelle ou la différence de valeur du véhicule dans lequel se fait le voyage}

Hacking (1998, pp. 65-89) expliquait que, si l'on ne comprenait pas les possibilités ambiguës offertes par la rupture de l'identité stable, on ne pouvait pas comprendre l'épidémie de personnalités multiples aux États-Unis. D'une part, la personnalité multiple répond aux célébrations postmodernes de la perte d'identité et, d'autre part, si on la considère comme une réponse à un trauma, elle possède les caractéristiques pathologiques liées à l'abus sexuel contre les enfants ${ }^{15}$.

${ }^{15}$ Les apports de Hacking sont, sur ce point, solidaires des analyses intéressantes faites par Paula Saukko (1999, pp. 41-42) au sujet de la manière dont la théorie de l'anorexie et de l'obésité, produite par une pionnière comme Hilde Bruch, reposait aussi sur deux préoccupations contradictoires. D'un côté, l'inquiétude face à un nouveau fascisme, popularisée par l'école de Francfort - Hilde Bruch était la disciple de Frieda Fromm-Reichmann, l'ex-femme d'Erich Fromm - et qui trouvera sa base dans le conformisme de la société de masses. De l'autre côté, la panique anti-communiste et sa référence à la perte de caractère indépendant des habitants de la vieille Amérique dans la société moderne. Les enfants suralimentés ou excessivement soumis aux autres constituèrent, dans ce contexte, deux figures psychiatriques surchargées de signifiés culturels, auxquelles Bruch prêta une allure «scientifique ». 
Pour engendrer des dispositifs de travail sociologique à partir du vecteur spécifié par Hacking, il est nécessaire de se demander comment une maladie peut, d'un côté, constituer un véhicule social attrayant pour un groupe de personnes, et de l'autre côté, comment elle peut condenser les signifiés négatifs d'un milieu culturel et social.

Je vais préciser ce que j'entends par «véhicule» au sens sociologique. Pour expliquer la trajectoire d'un individu, Bourdieu (1997, pp. 74-83) utilisait la métaphore du métro. Les agents sociaux se trouvent en un point du trajet; à partir de ce point, ils disposent d'une vision de leur avenir possible sous la forme de trajets alternatifs; cette vision conditionne les choix spécifiques de trajets faits par l'individu, tout comme ses rejets de certaines lignes de métro.

Passeron (1991, pp. 190-191) signalait que la perspective de Bourdieu néglige l'étude des véhicules dans lesquels voyagent les individus. Pour s'expliquer, il rappelle une image de Schumpeter : les classes sociales sont comme des autobus qui ne transportent pas toujours les mêmes voyageurs (les trajets d'autobus sont moins stables que le plan du métro, et de ce fait, ils représentent mieux la cartographie des destins sociaux). Ceci indique bien d'abord que les véhicules ne sont pas réductibles aux voyageurs qui y montent et en descendent, et ensuite que les véhicules changent de valeur sociale selon les caractéristiques des voyageurs qui y montent et en descendent.

Cette précision est nécessaire pour comprendre quels types de véhicules sociaux représentent la catégorie «anorexique » et la catégorie "boulimique ».

Julia est professeur d'université, fille d'une couturière et d'un policier municipal. Son grand-père paternel était pêcheur, le grand-père maternel maçon. Julia se considère, selon ses propres termes, comme issue «de classe moyenne inférieure ». Mais deux circonstances modifièrent le destin social de sa famille. Après l'arrivée massive de la gauche dans les conseils municipaux andalous, suite aux premières élections démocratiques, le père de Julia devient un des proches du nouveau maire de sa ville. De plus, Julia réside dans un quartier de classe moyenne. Après deux ans de surenchère corporelle, une amie de sa mère, épouse d'un psychiatre, la conduit au cabinet d'un psychiatre renommé. Julia connaissait déjà quelques livres sur l'anorexie :

Julia: Il m'a dit que j'étais anorexique. Je le savais parce que j'avais lu des livres. JLMP : Quel effet cela te faisait-il?

Julia: C'était une sensation un peu morbide. La sensation que nous étions bizarres, différentes...

JLMP : Spéciales... ?

Julia: Oui. C'était une chose qui arrivait à des femmes qui avaient un profil... intelligent, perfectionniste. Je me disais : c'est la vérité, je suis ainsi.

Dans un autre texte, j'ai montré comment le fonctionnement pratique de la distinction entre anorexie et boulimie, fonctionnement qui dérive pour une bonne part des descriptions faites par les spécialistes, comporte, par-delà des classifications cliniques que je ne mets pas en question, des projections sociales saturées 
d'ethnocentrisme de classe (Moreno Pestaña, 2005, pp. 130-137). La probabilité de mériter la classification de boulimique, si l'on provient des classes populaires, m'a paru beaucoup plus haute que celle de mériter la classification d'anorexique - sans que pour autant il y ait des différences dans les pratiques d'autocontrôle corporel employées par rapport à des patients provenant de classes privilégiées. Dans son analyse des classifications à l'œuvre dans un dispositif hospitalier, Helen Gremillion (2003, pp. 157-175) montre comment les stéréotypes sur l'anorexie, fondés sur des distinctions ethniques et de classe, divisent la population des utilisateurs. Ainsi, les patients issus des classes travailleuses ou d'ethnies non blanches sont classés comme borderline : les descriptions que font les spécialistes de leur comportement montrent la même tendance à naturaliser (et à stigmatiser), dans l'économie psychique de l'agent, deux règles de comportement, liées à l'origine populaire des patients borderline, que j'ai retrouvées dans mon travail de terrain, mais avec comme matrice la division anorexie / boulimie.

Ce dispositif discursif a surgi dans les matériaux recueillis au cours de mes entretiens. Ellen, une des personnes que j'ai interviewées, fille de professeur d'université, militante du mouvement gay, artiste et spécialiste des troubles alimentaires, m'a mis en rapport avec des gens qui disaient avoir souffert d'anorexie. Nadia, fille de professeurs d'université, militante de la cause homosexuelle et artiste, m'expliqua pendant l'entretien qu'elle s'était considérée comme anorexique après avoir entendu Ellen valoriser l'anorexie comme recherche de l'indéfinition corporelle et de la subversion des rôles sexuels. Nadia me mit en rapport avec une autre jeune fille, elle aussi artiste, qui se revendiquait comme ancienne anorexique. Dans l'immense majorité des récits que j'ai recueillis, les personnes, qu'elles aient été diagnostiquées ou non, furent capables de reconstruire une série d'événements qui permettaient de signaler, d'une part, l'existence de surenchères corporelles très intenses et, d'autre part, l'expérience de préjugés physiques et psychiques graves liés à celles-ci. Dans le matériau que j’ai recueilli avec Nadia, j’ai pu identifier, après quelques efforts, une séquence biographique de ce genre. Pour la dernière personne avec laquelle elle m'a mis en rapport, j'en ai été incapable. J'en conclus qu'il existe des espaces sociaux et professionnels dans lesquels s'avouer anorexique est une espèce de «stigmate » de privilégié.

Le côté positif de la polarité culturelle montre, d'une part, la logique de ce que Goffman appelait «stigmaphilie » 16 et, d'autre part, la «stigmaphobie » correspondante (cas de la boulimie et des patients anorexiques borderline). Par ailleurs, il montre comment les constructions du monde savant peuvent faciliter un processus de construction d'identité pour des gens situés à certains points d'un parcours social et professionnel. Parmi ces gens, il y a sans aucun doute des chercheuses qui, à partir

16 Goffman (1975, pp. 38 et 44) explique que les gens qui portent un stigmate «vivent dans un monde littérairement défini, aussi peu cultivés soient-ils. A supposer qu'ils ne lisent pas de livres consacrés à la situation de leurs semblables, du moins parcourent-ils des magazines et voient-ils des films : et même, à défaut de cela, ils ont toujours sur place des congénères doués pour la parole. Ainsi la plupart des personnes stigmatisées peuvent profiter d'une version intellectuellement élaborée de leur point de vue ». 
d'un capital d'identité (« je sais ce que je dis ») qu'offre le fait d'avoir éprouvé ce trouble, deviennent des participantes du marché concurrent du discours (le mien, par exemple) qui fleurit autour des troubles alimentaires ${ }^{17}$. Cette dimension contient donc des possibilités de promotion professionnelle et d'acquisition de capital symbolique que l'on peut faire jouer dans certains des milieux du monde de la production culturelle, y compris celui de l'université.

La conjonction des caractéristiques sociales de la population qui est passée par l'anorexie, leur certification dans un langage clinique sociocentrique et l'ouverture d'une large panoplie de discours à ce sujet, tout cela a ouvert pour ce trouble une nouvelle ligne de métro, pour reprendre l'image employée par Bourdieu, qui ne mène pas aux lieux les moins désirables de l'économie des biens symboliques - en modifiant une formule de Bourdieu (1988, p. 160), on pourrait dire qu'il n'y a rien de tel pour exalter un stigmate que de le recevoir d'un patricien. Ou, pour récupérer l'appréciation de Passeron, on a équipé un autobus qui, lorsqu'on le prend à partir de positions sociales favorables, permet un parcours distingué.

Quand les passagers de cet autobus proviennent des fractions de classes populaires que j'ai étudiées, le parcours ne mène pas vers les meilleures positions de l'économie des biens symboliques de leur milieu. Il y a une raison à cela: l'anorexie y est attribuée à des objectifs prosaïques. Juana, fille d'ouvrier qui habite une petite ville andalouse de moins de 20000 habitants, a été hospitalisée suite à un diagnostic d'anorexie. Malgré des origines sociales très modestes, Juana a reçu de son père une forte stimulation dans la recherche vers une mobilité sociale ascendante (elle poursuit aujourd'hui des études d'ingénierie informatique). Quand elle entama son parcours, l'anorexie ne symbolisait pas pour elle une qualité extraordinaire quelconque:

Je voyais ça (comme une chose) de filles stupides qui veulent devenir mannequins, et tout le monde ne va pas viure de son physique, non? Pour moi, les anorexiques étaient des ingénues de la vie, peu réalistes. Je pensais : "Ma fille, accepte-toi. Tout le monde a ses bons et ses mawvais côtés».

Yolanda, fille d'ouvrier qui vit dans une ville de 10000 habitants suivait, au moment de l'entretien, un traitement contre l'anorexie depuis plus ou moins cinq ans. Son patelin, selon elle, était " plein d'anorexiques ». La cause en est, à ses yeux, tout à fait vulgaire : "Il y a beaucoup de connerie. Il y a beaucoup d'argent et les gens aiment bien frimer».

Les investissements corporels considérables sont associés, dans certaines fractions des classes populaires, à un désir de visibilité sur le marché sentimental et

17 Le cas de Martha McCaughey (1999, pp. 133-155), docteur en sociologie de l'Université de Californie à Santa Barbara et professeur de Women's Studies en Virginie, semble parallèle à celui d'Ellen. Comme cette dernière, elle explique que son anorexie a commencé en réponse au harcèlement sexuel de quelques jeunes gens; de plus, l'anorexie a une fonction de subversion des rôles sexuels et ne dépend pas du tout, comme le signalent d'autres théories, d'une complaisance vis-à-vis du regard masculin. Enfin, la recherche de l'invulnérabilité est l'effet que proposent les techniques d'autocontrôle corporel. Une des manières pour la sociologie de préciser les dimensions de ce vecteur est d'analyser les modalités d'autoconfession par rapport à l'anorexie. Je le ferai dans un autre travail. 
matrimonial, qui n'est légitime que dans une certaine classe d'âge. Un groupe de discussion composé de jeunes élèves de lycée ${ }^{18}$ dans un village de 10000 habitants circonscrit le désir de maigrir à la recherche d'un petit ami et/ou d'un mari. Une fois cette recherche aboutie, la préoccupation de la corpulence diminue:

- Je crois que quand (une femme) va se marier, tout cela (la pression d'être attirante) lui est déjà plus égale.

- Et quand tu as déjà un petit ami? Parce que j’ai déjà un petit ami, et ça m'est déjà égal, je l'ai déjà attrapé (...).

$-($ Rires $)$

- C'est parce que quand tu as un petit ami, tu t'en vas manger dans les bars tous les soirs, et là déjà tu grossis forcément (...).

-Quand tu sais déjà que tu tiens ton mec, sûr, tu ne te préoccupes pas tant de (l'apparence physique).

Olga, 31 ans, fille d'un propriétaire de restauroute, sans étude, mariée et vivant avec quatre enfants, dont deux du précédent mariage de son mari, gérant d'un petit supermarché, associe ses troubles alimentaires à la peur de grossir après le mariage. Dans son cas, le mariage ne fonctionne pas comme un symbole positif de la distension corporelle, comme dans les références antérieures, mais bien comme un possible «funeste » dont elle veut se protéger par son autocontrôle corporel. Olga a cinq sœurs, toutes «obèses » selon elle, qui lui rappellent toujours le moment où disparaîtra son charme physique: "Mes seeurs me disaient toujours: tu verras quand tu te marieras! Là tu grossiras comme nous!, j’ai toujours voulu éviter ça».

Comme on peut le vérifier, il est plus difficile dans les classes populaires de trouver, associées à l'anorexie, des valeurs distinctes de celles de la recherche de la visibilité sur le marché matrimonial et sexuel. La possibilité d'associer des caractéristiques sublimes ${ }^{19}$ à l'anorexie semble augmenter avec le niveau social et l'appartenance aux secteurs les plus dotés de capital culturel d' «avant-garde ». On peut extraire de mes entretiens trois formes fondamentales de «culte » de l'anorexie. D'abord, l'anorexie identifie une population dotée d'attributs d'excellence: les mots de Julia, parmi d'autres que j'ai recueillis et qui allaient dans le même sens, en sont un exemple. Ensuite, l'anorexie est liée depuis longtemps à un modèle de beauté morbide en marge de la norme dominante, mais non moins institutionnalisé pour

18 Filles de pères ouvriers ou agriculteurs, sauf dans un cas, où le père est agent commercial, avec une grande majorité d'études primaires, et deux cas où soit le père soit la mère a fait des études secondaires.

19 Il s'agit là d'un discours qui a une grande influence sur certains spécialistes des sciences sociales qui prétendent fournir une vision «non simplifiée » de l'anorexie. Selon Maître (2000, p. 25), c'est pour la raison suivante qu'il faut séparer l'anorexie de la recherche de la beauté : "Puisqu'il existe un large accord pour attribuer à l'anorectique une angoisse devant le risque d'être une femme sexuellement attirante, on ne voit pas en quoi ce sentiment pourrait motiver le désir de se conformer aux canons de la séduction érotique». 
autant, dans le menu des possibles féminins ${ }^{20}$. Rosana, en traitement contre l'anorexie depuis trois ans environ, fille d'un ouvrier devenu grand entrepreneur sur la côte andalouse, considère que l'anorexie offre quelque chose de «singulier » :

Ça me plaît de me sentir fragile. Je sais que ça ne plầt pas aux mecs, mais ça m'est égal. Me trowver un peu fragile, avec la peau sur les os, ça me donne plus de sécurité, je ne sais pas, de beauté. J'aime l'autocontrôle, le sens de la fragilité. Et pourquoi pas, être malade.

En troisième lieu, l'anorexie peut caractériser un modèle de subversion des rôles sexuels associé, dans certains cas, à un projet radical d'innovation corporelle à prétentions artistiques. Dans l'univers artistique, la vie individuelle, conçue en certaines occasions comme la construction d'une légende, est un instrument fondamental pour la valorisation des agents.

Aucun de ces trois critères d'exaltation de l'anorexie n'est apparu dans les matériaux recueillis auprès de mes sujets d'origine populaire. En ce sens, il faudrait corriger Hacking. Les catégories psychiatriques pourraient être décrites comme des autobus dans lesquels voyagent les individus: selon ses origines sociales, sont attribués à la population qui y monte des signifiés qui sont distincts des véhicules. Les parcours que ceux-ci offrent sont ainsi plus ou moins avantageux selon les marchés sociaux dans lesquels les sujets évoluent. On ne peut donc pas parler d'une polarité culturelle unique pour l'ensemble d'une société.

$\mathrm{Ou}$, en tout cas, pas encore. Aujourd'hui, quand quelqu'un entre dans la catégorie « anorexie » à partir d'un milieu modeste, il ne bénéficie pas de l'esthétique de l'anorexie, présente dans les catégories favorisées. Le pôle positif de la polarité culturelle demeure très faible dans les classes populaires. De plus, comme je l'ai indiqué plus haut, les modèles de comportement caractéristiques des classes populaires n'aident pas à la classification par les spécialistes comme de véritables anorexiques. Dans la vie quotidienne des classes populaires, la désignation manque d' "aura " (je reviendrai plus loin sur cette question cruciale), alors que pour les spécialistes, les origines populaires ne facilitent pas l'obtention de la désignation

20 « La belle malsaine et le désir qui lui est associé hantent toutes les époques de la culture, connaissant bien sûr différentes amplitudes. Parallèlement à son apogée avec le XIX ${ }^{e}$ siècle, cette esthétique d'un corps malade (ou, à tout le moins, malsain) se prolonge aujourd'bui dans de nombreux domaines de la culture, arts plastiques ou cinéma, et tout particulièrement dans la mode. Elle s'inscrit, comme elle l'a toujours fait, 'à rebours' du modèle dominant : au XIX ${ }^{e}$ siècle, le modèle de la beauté phtisique et nerveuse s'inscrit en réaction au modèle dominant de la bourgeoisie grasse et sédentaire et, aujourd'bui, certaines images d'une beauté maladive s'inscrivent en rupture par rapport au modèle dominant du corps accompli, mince, lisse et tonique. (...) La belle folle, si elle est moins contagieuse que la belle malsaine, n'en est pas moins désirable. Un bon exemple de ce lien entre déséquilibre nerveux et état physique esthétisé se trouve à l'évidence dans l'anorexie, maladie féminine typiquement contemporaine dont de nombreuses expressions sublimées se retrouvent à intervalle régulier dans l'esthétique de la photographie de mode, rejoignant celle de la beauté souffreteuse évoquée plus haut. L'anorexie est également exemplaire de la manière dont notre époque relie une expression trouble de la beauté à un déséquilibre, physiologique comme nerveux, par ailleurs relié, comme la mélancolie, à l'intelligence ». Remaury (2000, pp. 67 et 71). 
«anorexie » ${ }^{21}$. Ce seuil d'entrée dans les troubles alimentaires est plus difficile à franchir quand on vient des classes populaires.

\section{Des déviances rapidement découvertes}

La constitution d'une niche écologique pour une maladie mentale transitoire exige que l'on puisse identifier les comportements «pathologiques ». De fait, l'une des maladies étudiées par Hacking, l'épidémie de fugue, n'a pas été diagnostiquée aux États-Unis en raison du manque de contrôle par l'État des mouvements de population. En France, en revanche, la conscription militaire obligatoire imposait aux citoyens d'informer les autorités de leurs mouvements. Il devenait donc possible de détecter les individus à inclure dans l'épidémie de fugue, alors qu'aux États-Unis, ils restaient inobservables, mêlés aux autres mouvements de population impulsés par la culture états-unienne et son «Go West, young man » (Hacking, 2002, p. 143).

Je tirerai de ce vecteur une question sociologique distincte de celle qui structure l'analyse de Hacking. Plutôt que d'étudier les dispositifs étatiques de visibilité et de contrôle, je me concentrerai sur la manière dont la diversité de culture somatique entre les groupes sociaux impose des processus différents de visibilité des déviances (Darmon, 2003, p. 181).

Dans mon travail de terrain, la possibilité d'être désigné comme déviant à cause d'une surenchère corporelle m'a paru spécialement forte dans certaines fractions des classes populaires. Je présenterai deux cas dans lesquels nous observons: i) une configuration familiale d'origine très éloignée des normes somatiques dominantes; ii) une intériorisation de règles somatiques en contradiction avec les objectifs corporels désirés ; et iii) une visibilité particulièrement intense de toute surenchère corporelle, ce qui facilite sa désignation comme déviante.

\section{Une reconversion somatique épineuse}

María José (30 ans, actrice) est née dans une ville de taille moyenne de la côte andalouse; elle est fille d'un petit fonctionnaire qui n'a pas fait d'études et d'une femme au foyer, et petite-fille de paysans de niveau économique très modeste. Parmi les clés de sa trajectoire, María José distingue une relation à l'avenir marquée par la peur du risque. Cette relation au futur procède, selon elle, d'une forte influence de la religion sur son éducation. Celle-ci se manifestait par une propension à vouloir aider les autres (María José se souvient qu'elle désirait devenir sœur missionnaire) et la conscience que la vie terrestre était chargée de souffrances:

${ }^{21}$ Il convient de préciser qu'il y a diverses fractions parmi les spécialistes. Selon mes observations de terrain, ceux qui fonctionnent comme fabricants de morale des troubles alimentaires ont tendance à faire un usage inflationniste des catégories: l'anorexie serait partout, sans distinction d'âge, de classe sociale ou de sexe. Dans certains cas, cette utilisation inflationniste s'accompagne de discours fondés sur les racines biologiques de l'anorexie. Cette indication introduit une question qui exigerait un développement beaucoup plus ample. 
(Je désirais) faire tout mon possible pour que le chemin (de la vie) t'amène au ciel. Et si ce chemin t'apportait des souffrances, (il fallait continuer à le suivre), contrairement au chemin de roses, qui était celui qui te conduisait directement à l'enfer (...) Le chemin sur la terre était épineux, c'était le chemin que je désirais, je désirais souffrir.

La conscience de la souffrance se conjugue avec des moments où des possibilités de sortir de son milieu s'ouvrent à elle. L'un de ces moments se produit lorsqu'elle entre au lycée. Là, elle se lie à des gens socialement différents et culturellement stimulants :

Au lycée, tout ce besoin de moyens spirituels se mêla en moi avec le (désir) d'acquérir un statut social. Je me rappelle un jour où nous sommes allés à la montagne, pour la voir, pas pour skier ni rien, et quand j'ai vu cela, quand j'ai vu le monde qu'il y avait là-bas, qui était tellement différent de mon quotidien, tellement artificiel et en même temps tellement... (expression d'admiration), je me disais : je dois étudier ce qu'il faut pour arriver à cela (...), je désire être, je désire avoir ces possibilités, et une fois que je serai là, prendre les mesures nécessaires ou me rebeller pour ainsi dire... Je crois que ce jour-là, je ne sais pas quel jour c'était, j'ai décidé que je voulais étudier la médecine.

Au lycée, María José commence à se préoccuper de son alimentation. Dans sa famille, qui s'apparente, à cet égard, aux fractions des classes populaires les moins atteintes par les préoccupations esthétiques, sa mère était, disait-elle, "obsédée » par le désir d'alimenter abondamment. "La nourriture, chez nous, est toujours liée à la protection et à la chaleur... C'est toujours la nourriture qui nous réunit». Tout en se préoccupant de son alimentation, María José n'a jamais prétendu changer les modèles alimentaires de sa mère. Elle est extrêmement lucide sur la manière dont son milieu familial lui rendait difficile la compétition corporelle avec ses compagnes qui provenaient d'autres configurations familiales, dans lesquelles la préoccupation du régime revêtait un caractère plus officiel :

(Il y avait des filles) qui finissaient par avoir une obsession de la nourriture, mais comme plus officielle. Leurs mères les mettaient au régime et... «tu verras avec le régime de la pêche, tu perds deux kilos en une semaine ». (...) À ce moment-là, j’avais le contrôle sur ma nourriture, mais de manière normale. Par exemple, je n'achetais pas de revues de régime et ... je n'avais peut-être pas de problèmes avec les vêtements que je mettais. En été, par exemple, je mettais n'importe quoi qui m'amusait.

Malgré le caractère non officiel de sa régulation, María José a réussi à réduire son poids et à se fabriquer pendant un certain temps une silhouette à sa guise. Cet autocontrôle n'a pas réussi à se stabiliser, mais constitue un moment-clé dans la longue histoire de ses surenchères corporelles discontinues. Quand on les analyse de manière diachronique, il semble qu'elles aient produit d'abord un sentiment de réussite, ensuite une espèce de "mémoire du corps » qui peut être réactivée dans un contexte différent. La disposition à l'autocontrôle corporel n'a certes pas été intériorisée : surtout, du fait de l'incapacité à modifier un milieu familial de manière à y faire fonctionner cette disposition de manière officielle, c'est-à-dire reconnue comme légitime. Néanmoins, le succès momentané et l'expérience corporelle de l'autocontrôle persistent comme un possible atteint: l'individu s'est prouvé que modifier son corps n'était pas simplement un désir, mais aussi une possibilité 
pratique. Une possibilité vécue, appropriée de manière perceptible, qui laisse sa marque sur les sensations du corps. María José ne stabilisa pas sa silhouette, mais elle se rappelle qu'à l'époque de son autocontrôle juvénile, elle avait commencé à admirer sa clavicule et à lutter contre la faim.

María José entama des études de médecine et les abandonna lorsqu'elle fit la connaissance d'un groupe de théâtre, qui lui montra sa véritable «vocation ». À ce moment-là, les règles alimentaires familiales devinrent une menace par rapport à ses objectifs corporels. Chaque fin de semaine qu'elle passait au domicile familial, María José craignait de grossir: la préférence familiale pour une cuisine bien préparée et pour la viande se conciliait mal avec sa volonté d'ingérer des aliments légers et de cuisiner sans trop d'assaisonnement. Ses pratiques alimentaires se poursuivirent sans pour autant devenir «officielles ». Pour les rendre telles, elle aurait dû, d'une part, lutter avec sa mère, ce qui aurait contredit sa tendance, selon elle centrale, à se mettre à la place des autres. D'autre part, les goûts alimentaires de la famille étaient intériorisés par María José : lutter contre l'alimentation maternelle revenait donc à lutter contre une partie d'elle-même.

María José obtint une bourse pour aller étudier dans une école d'art dramatique. C'est le moment où elle se sépare de sa famille, le moment aussi où elle commence à vomir. Dans son discours, manger est lié à la chaleur; vomir en revanche se rapporte au froid, à la liberté et à l'acceptation du risque. Dans son expérience concrète, vomir représente une forme de compromis entre deux exigences: une relation incorporée à l'égard des aliments, renforcée et maintenue par la vie familiale, et une panique très forte face à l'effet que cette relation produit sur son corps. La nourriture est une " espèce de monstre, un Alien ou quelque chose de ce genre, qui se met en toi, ça te fait paniquer, ça te possède d'une certaine façon... » ${ }^{22}$.

Au sein de cet espace domestique, María José n'a pas trouvé de lieu pour son projet corporel. Elle l'a trouvé en revanche dans son propre corps, mais en le faisant coexister avec les goûts qu'elle avait incorporés dans son domicile familial. Dans sa famille, le corps officiel entrait en conflit avec le corps officiel du monde que María José désirait intégrer: dans sa mémoire, ceci est en rapport avec une décision mythique, celle qu'elle prit le jour où elle gravit la montagne. Au moment de notre entretien, elle avait déjà décidé quel monde elle préférait. Mais dans ce monde, dans le Nostromo (un cargo spatial dans lequel l'équipage rencontre le «huitième passager ») dans lequel elle désirait voyager avec une fraction des classes moyennes, sa classe d'origine restait présente sous forme de «monstre » qui menaçait de la posséder à travers l'envie de manger.

22 Bourdieu (1992, pp. 173-174) partageait avec María José l'opinion que le monstre créé par Ridley Scott était un bon exemple des clés sociales de l'angoisse personnelle. Répondant à Loïc Wacquant sur le dispositif de recherche qui allait donner lieu à La Misère du monde, il signalait ceci : «Nous procédons à des entretiens très approfondis visant à aider les personnes interrogées à découvrir et à livrer les principes profonds de leurs drames extrêmes ou de leurs malheurs ordinaires; à leur permettre de se débarrasser de cette réalité extérieure qui, à la façon du monstre Alien, les babite et les hante, et les tient de l'intérieur, les dépossédant de l'initiative de leur existence. Alien est une sorte de mythe moderne qui offre une bonne image de ce que l'on appelle l'aliénation ; c'est-à-dire de cette présence de l'altérité au cour de la subjectivité ». 
Ces deux mondes impliquent des modèles corporels contrastés. Chacun de ces modèles divise l'ensemble des scènes dans lesquelles se déploie la vie de María José. Des scènes qui représentent, d'une part, sa classe d'origine et, d'autre part, sa classe de destin dans laquelle elle évolue de manière quotidienne, sans toutefois s'y être absolument installée : une disposition puissante de son propre corps le lui rappelle à la moindre défaillance.

Lorsque l'on est issu de fractions des classes populaires semblables à celle de María José, toute régulation corporelle est sanctionnée. Dans son cas, c'est elle-même qui opère cette sanction : elle sait que c'est la valeur du travail de sa mère qui se verrait déniée si elle rejetait sa nourriture. La pluralité de projets corporels n'a pas sa place dans l'économie symbolique de la famille: peut-être en va-t-il de même dans l'économie budgétaire, vu que l'homogénéité du menu peut aussi être un impératif dicté par la modestie des moyens financiers. Dans ce contexte, une surenchère corporelle sera facilement perçue comme déviante. Cette perceptibilité peut mobiliser un processus de correction qui peut facilement tirer parti de l'usage profane des étiquettes qui popularisent les catégories cliniques de l'anorexie et de la boulimie.

\section{Les censures dans la recherche de la beauté}

J'ai connu Olga grâce à Pedro, son mari. Celui-ci s'est rendu à l'association d'aide aux anorexiques pour parler de sa femme, alors internée dans une clinique. Le mari d'Olga (42 ans) est une personne forte et corpulente qui vous regarde dans les yeux au cours de la conversation et qui n’hésite pas à demander à la psychologue de mieux s'expliquer: "Je suis un bosseur », explique-t-il. Pedro se plaint amèrement de l'anorexie de sa femme. C'est son second mariage et il dit ne pas souhaiter «se charger d'une personne immature qui ne sait pas jouir de la vie. Elle ne veut pas vivre dans un petit village, mais j'ai beaucoup travaillé pour avoir ce que j'ai. Mais bon, c'est la mère de mes enfants. En plus, l'bospitalisation m'a coûté un paquet d'argent». Je suis allé voir Olga dans le village où elle habite. Olga travaille au supermarché dont son mari est gérant. Il ne m'a pas fallu beaucoup d'efforts pour identifier Olga. Parmi les autres travailleurs, elle détonnait par son aspect: des cheveux courts teints en bleu, un pantalon collant et une définition corporelle plus proche de celle des jeunes étudiantes d'une ville universitaire que de mon propre stéréotype de la femme mariée avec deux enfants vivant en milieu rural.

Nous entrons dans la maison d'Olga et je réalise l'entretien avec elle et son mari. Olga est très nerveuse et la conversation très tendue. Elle regarde beaucoup Pedro, lequel accompagne de soupirs désapprobateurs toutes les questions se rapportant aux pratiques alimentaires d'Olga. Après l'entretien, dans une ambiance beaucoup plus décontractée, ils m'invitent à manger. Pendant qu'Olga prépare le repas, Pedro me fait visiter la maison, les terrains qu'il a acquis et me montre ses chiens. Puis, ils me demandent ce que j'ai l'habitude de manger, Pedro me regardant d'un air ironique en disant: "tu manges normalement? ", suggérant que j'étais trop mince pour un homme de mon âge. Olga ne mange pas la même chose que Pedro et les quatre enfants : elle évite les graisses et se contente d'une grande salade. 
J'ai expliqué plus haut qu'Olga avait peur que son mariage ne la fasse grossir. Une fois mariée avec Pedro, Olga a commencé à se trouver grosse quand elle allait dans les magasins de vêtements. Elle a commencé à restreindre son alimentation, ce qui a déclenché les reproches de Pedro : «Elle ne mangeait pas la même chose que nous et je pensais qu'elle suivait un régime ». À la maison, le comportement d'Olga a vite été signalé comme déviant: il s'agit du cas embarrassant d'une mère qui donne à ses enfants ce dont elle ne veut pas pour elle-même. Notons qu'Olga n'était pas en mesure d'imposer sa norme diététique dans la famille. Tout au contraire, les repas en famille plaçaient Olga dans des situations angoissantes, aussi bien avec la mère de Pedro qu'avec lui-même et les enfants. Aller manger dans un restaurant chinois avec sa famille, par exemple, faisait une peur terrible à Olga. Pendant toute la conversation, elle n'a pas eu recours à un seul argument de santé pour défendre ses pratiques alimentaires. À un certain moment, je lui ai demandé si quelqu'un lui avait fait remarquer qu'elle avait perdu du poids. Sa réponse est très significative de l'« illégitimité » qu'Olga associe à ses motivations pour restreindre son alimentation :

Je n'ai jamais eu de problèmes. Peut-être, quand j'étais jeune, quelqu'un m'a dit: "quelles jambes, tu attrapes!". (Elle baisse la voix), "Mon Dieu, je ne vais plaire à personne! ». (Elle reprend son ton normal) Tout est très compliqué.

La recherche de la beauté implique une deuxième pratique déviante. Si le mariage « fait grossir », c'est parce qu'il suppose la fin de la tension pour la visibilité sur le marché sexuel. La préoccupation corporelle d'Olga semble signifier qu'elle n'a pas renoncé à tous les possibles sur ce terrain. De la sorte, Olga devient déviante par rapport aux définitions dominantes des classes d'âge dans son milieu social: quand elle va dans les magasins de vêtements, Olga choisit toujours des tailles plus petites que sa fille; c'est pourquoi elle ne cherche pas dans les vêtements de sa tranche d'âge, mais dans ceux des franges adolescentes.

Tout milieu social offre un catalogue de déviances légitimes et illégitimes. Il faut les esquisser si l'on veut comprendre comment les cultures de certains groupes et fractions de classes permettent certaines pratiques déviantes et en interdisent radicalement d'autres. Quand Olga se réfere à ses pratiques anorexiques, elle les fait dépendre de sa consommation de cocaïne. Cette drogue que consomment Olga et Pedro " fait beaucoup maigrir », me dit-elle. La cocaïne permet de ne pas manger pendant des jours (Olga donne trois jours comme maximum). Un des effets de la cocaïne - la perte d'appétit - a été codifié dans le cadre des pratiques de régulation corporelle: en même temps, Olga rejette le haschisch qui augmente l'appétit. Par ailleurs, la cocaïne pouvait être présentée publiquement dans le contexte quotidien d'Olga.

Ni Olga, ni María José n’ont pu inclure leurs pratiques corporelles de manière publique dans un segment fondamental de leur contexte quotidien. De ce fait, elles ont mis en œuvre des pratiques déviantes : toutes deux ont employé le vomissement, Olga a utilisé la cocaïne en plus. De cette manière, elles tentent de contrôler les effets corrosifs que leurs modèles corporels introduisent dans leurs configurations familiales respectives. Après avoir été classée comme anorexique, Olga a obtenu une certaine compréhension de son mari. "Je ne savais pas que c'était une maladie", m'a expliqué Pedro. Olga a ainsi évité l'accusation d'être une «mauvaise » mère (qui 
donne à ses enfants ce dont elle ne veut pas pour elle-même) et une "mauvaise " épouse (qui n'a pas mis fin à sa recherche de visibilité corporelle et qui n'a pas accepté la sentence que «le mariage fait grossir»). Depuis lors, Olga mange, en tant que malade, un menu différent de celui de sa famille.

Dans les deux cas, la rupture avec la culture somatique du groupe familial empêchait un autocontrôle corporel officiel (dans les classes populaires analysées, une telle rupture est légitime à l'intérieur d'une certaine classe d'âge, pourvu qu'elle soit temporaire). D'une part, du fait qu'elles ne sont pas officielles, ces pratiques corporelles donnent lieu à des comportements plus déviants que ceux dérivés d'un régime alimentaire et corporel public et légitimé ${ }^{23}$. D'autre part, le souci de la silhouette corporelle suppose le questionnement pratique du principe de conformité avec le groupe. La conjonction de ce principe de conformité et de pratiques de restriction extrêmes produit, dans un premier temps, des alertes rapides face à n'importe quelle déviance, puis compte tenu des modes de régulation de la corpulence employés, la possibilité de les qualifier de pathologiques. Si ce processus de stigmatisation de la déviance obtient l'appui d'une catégorie médicale (ce qui est de plus en plus facile), on peut aboutir rapidement à un circuit institutionnel de gestion de la maladie. Dans ce type de configuration familiale, il est facile de penser que le seuil représenté par le vecteur de la visibilité est très ouvert.

\section{Les conditions sociales d'impossibilité des troubles alimentaires. Comment se défendre de la contrainte esthétique tout en ne l'oubliant pas?}

Dans son analyse de l'épidémie de fugue, Hacking explique comment l'origine sociale des agents étudiés a fourni de nombreuses pistes sur l'une des composantes fondamentales des niches écologiques. Les gens qui avaient «pris » le chemin de la fugue étaient situés à des positions ambiguës dans l'espace social. Ils n'étaient pas vagabonds (on ne peut pas fuguer si l'on n'a pas de domicile), ni paysans ou fermiers; ils ne travaillaient pas non plus en usine, mais cela ne faisait pas d'eux, pour autant, des membres des classes moyennes ou supérieures. Les individus qui «fuguaient» étaient des travailleurs disposant d'une certaine autonomie (employés du chemin de fer, petits commerçants, charpentiers, verriers, tailleurs ou cordonniers), incapables de gérer leur vie quotidienne et désireux de s'en échapper. Pour cela, ils ne disposaient que de l'issue que «propose » la maladie mentale, laquelle leur concédait ainsi une

23 Yolanda explique qu'elle n'avait jamais pu obtenir un plat spécifique chez elle, avant d'être diagnostiquée anorexique. Pour maigrir, elle a donc fait du sport et arrêté de manger. Elle argumente qu'elle a appris à «bien » manger - c'est-à-dire à manger sans grossir - dans les activités de l'association d'aide contre l'anorexie. De cette manière, elle a acquis la norme diététique légitime qu'elle a réussi à imposer chez elle, au moins pour elle-même, grâce à la légitimité qui vient du fait d' «être malade ». Dans le groupe de discussion composé de jeunes universitaires provenant des classes populaires, "cesser de manger» apparaissait comme la pratique de restriction à la fois la plus irrationnelle et la plus répandue. 
liberté qui relâchait leur souffrance quotidienne (Hacking, 2002, pp. 115-117). La fugue donne à ces travailleurs la possibilité de faire ce que font les vagabonds, sans obligations sociales, ou les bourgeois qui se détendent grâce au tourisme.

À ce moment de son argumentation, Hacking énonce un des points sensibles de toute approche sociologique de la maladie mentale: quelle pertinence peut avoir le recrutement sociologique des personnes encadrées par les dispositifs de la maladie mentale? En ce qui concerne les troubles alimentaires, dans quel lieu de l'espace social est-il possible de développer des pratiques susceptibles d'être considérées comme anorexiques ou boulimiques? Ces pratiques de surenchère corporelle sontelles susceptibles de se réaliser en tout point de la géographie sociale ? Ou l'inégalité dans la dotation de capitaux permettra-t-elle, à certains groupes, de les réaliser et à d'autres, non?

Muriel Darmon, dans un travail que je trouve fort riche, s'est risquée à répondre. Mais, selon moi, elle n'a pas pris pour le faire le meilleur des chemins. Dans son dispositif d'analyse, les données épidémiologiques en matière d'anorexie (prédominance féminine, âge adolescent et origines sociales moyenne et supérieure) sont prises comme description des propriétés structurales typiques de la population. Ensuite, à partir d'un corpus d'entretiens socialement homogènes (classes moyenne et supérieure) dont les conclusions sont analysées fondamentalement à partir de la cartographie des goûts, utilisée par Bourdieu dans La distinction, elle considère que les anorexiques constituent une espèce de condensation psychosociologique de leur lieu dans l'espace social : compétitivité, obsession du statut représenté par le corps et la culture, etc. De cette façon, Darmon (2003, p. 249) se propose de dessiner «les conditions sociales de possibilité, nécessaires mais non suffisantes » de l'activité anorexique. De fait, avec un dispositif de raisonnement sociologique qui inclut, d'abord, l'adoption non critique des données épidémiologiques, puis un corpus d'entretiens découpé sur la base de ces données et, enfin, une ouvre sociologique de référence fondamentalement construite à partir d'une théorie des grands capitaux (ce qui est le cas de La distinction ${ }^{24}$ ), on ne voit pas comment pourrait être corrigé le cadre de départ (configuré par des données épidémiologiques confirmées spéculativement par un dispositif d'entrevues et une ouvrage théorique).

En ce qui concerne les classes populaires, Darmon renvoie à de vastes thèses sociologiques qui considèrent que, dans les classes populaires, la recherche de la distinction corporelle n'a pas cours dans le quotidien. Certes, ces thèses incluent une part de vérité : sur le terrain corporel, les classes populaires referment leur univers de possibles plus tôt que ne le font les classes moyenne et supérieure. Mais cela ne signifie pas qu'ils l'oublient totalement. Comme je l'ai signalé dans le cas de María José, une expérience corporelle gratifiante peut se perpétuer comme un passé qui interpelle encore le présent et qui peut, dans certaines occasions ou circonstances, être réactivé dans l'avenir. Si l'on oublie cela, on a tendance à identifier la fermeture des possibles corporels avec les pratiques de deuil, nécessaires pour oublier les modèles

${ }^{24}$ Il me semble que le concept de «misère de position ", employé par Bourdieu dans La misère du monde, aurait permis un autre cadre de raisonnement sociologique. 
corporels abandonnés. Les classes populaires se pensent d'une manière fort pauvre: pour reprendre une belle formule d'Olivier Schwartz (2002, p. 484), nous nous mettons à "les imaginer sans imaginaire ». Toutes les opérations mythiques qui permettent au misérabilisme (les corps populaires seraient de simples réserves de force de travail) de se transformer en populisme (la distinction et ses angoisses seraient extérieures au monde populaire, un monde "sain » et libéré de ce que Pier Paolo Pasolini appelait l'« infection » de la vie bourgeoise) commencent à s'implanter dans l'analyse 25 .

La relation des dominés aux normes corporelles légitimes peut se décliner de diverses manières. Entre l'incorporation de la norme et son oubli, il existe des formes de ce que Passeron (1970, p. 23), commentant une idée de Hoggart (1970, p. 49), appelle «assimilation à éclipses ». Utilisant le matériau extrait des groupes de discussion, je montrerai comment cette «assimilation à éclipses » peut être repérée dans l'existence de quatre principes pratiques de réduction de la tension corporelle. Dans la mesure où ces principes s'articulent dans un groupe social, la tension corporelle se relâche nettement et, de ce fait, les troubles alimentaires, dérivés de restrictions alimentaires sévères, se transforment en impossibles. Dans cette analyse, je m'appuierai sur le matériau produit dans les groupes de jeunes élèves de lycée. Un groupe à la fois féminin et juvénile: les deux traits (tension liée aux marchés du genre et tension liée à un moment d'ouverture maximale sur le marché sentimental et sexuel) le rendent sensible aux modèles corporels légitimes. Si ces jeunes n'oublient pas les principes que ces modèles irradient, il ne semble cependant pas que leur existence soit organisée complètement par eux. Ces conditions d'impossibilité ne sont pas définitives. Les sujets qui pratiquent ce que l'on pourrait appeler «contrôle du contrôle corporel » peuvent s'avérer défaillants dans son application. De plus, ces principes ne fonctionnent que dans des contextes collectifs : en d'autres termes, c'est seulement si les individus avec lesquels on partage la vie quotidienne les pratiquent que l'on peut les incorporer sans risquer de souffrir d'une dépréciation corporelle.

Somme toute, ces principes corporels constituent les traits de base de ce que, suivant Bourdieu (2002, pp. 144-151), j’appellerai les marchés corporels «francs » au sein des classes populaires. Selon Bourdieu, les individus qui appartiennent à un groupe social éprouvent la pression de divers marchés : les uns, les marchés francs, dans lesquels la valeur des produits (dans notre cas, des produits corporels) est définie selon des critères internes à la classe sociale; les autres, les marchés tendus, dans lesquels la définition de la valeur des produits corporels exige la reconnaissance de la norme dominante et, donc, la recherche anxieuse de la correction ou, si c'est impossible, la protection de l'invisibilité corporelle. Certes, un marché franc, dans la mesure où il peut être colonisé par les marchés dominants (constitués, pour ceux qui ne contrôlent pas leurs principes, comme marchés tendus), peut cesser d'exister. Ainsi, son maintien exige l'engagement des agents qui y sont intéressés et la

25 Sur l'idéalisation romantique des familles populaires (d'où proviennent les anorexies atypiques et borderline) et sur sa coexistence avec la stigmatisation de ces mêmes familles, voir Gremillion (2003, pp. 186-187). 
stigmatisation de ceux qui désirent l'altérer (point que je ne développerai pas ici). Il me semble possible de dégager quatre principes dans ce type d'engagement ${ }^{26}$.

a) Principe d'activation située de la surenchère corporelle. Il importe de comprendre que, pour ce qui concerne la tension corporelle, les marchés francs et les marchés tendus se combinent dans l'expérience vitale des gens. Le mépris des modèles corporels légitimes menace les individus des classes populaires d'une espèce de mort sociale: l'absence de visibilité sur le marché sentimental et sexuel. C'est pourquoi, le matériau obtenu dans mon travail de terrain, plus quà un abandon définitif de la tension corporelle, fait penser à une composition tendue des deux types de marchés. Selon qu'on participe à l'un ou à l'autre, on s'attache plus ou moins aux principes légitimes se rapportant au corps. La métaphore de l'«assimilation à éclipses " prend ici tout son sens. Un exemple, à ce propos, montrera la diversité selon les saisons. Ainsi, dans le groupe de jeunes élèves de lycée, on disait ceci :

- Que vienne l'été et toutes au régime d'été: "il faut se mettre en bikini!".

- En biver, comme tu es plus couverte, tu peux dissimuler.

- En été, tu vas au gymnase pour pouvoir montrer tes jambes.

- En biver, comme on ne voit rien, tout le monde s'en fiche.

- b) Principe de limitation du travail sur le système des goûts. L'analyse réalisée sur la trajectoire de María José montre de quelle manière les goûts alimentaires familiaux et les modèles corporels désirés coexistent jusqu’à faire chanceler sa vie intime. Dans le groupe de discussion auquel je viens de me référer apparaissait une autre possibilité : des aliments diététiques peuvent être incorporés dans le régime alimentaire, dans la mesure où ils peuvent s'accorder au système des goûts acquis auparavant ${ }^{27}$. Ce principe de limitation du travail sur le système des goûts inclut aussi un principe d'accord avec le système des goûts du groupe domestique :

- Moi, je crois que les goûts, si quelque chose te plaît, même si tu fais un régime, tu vas le bouffer. En fait, le régime, tu le laisses tomber.

- JLMP : Alors, on ne peut pas changer les goûts?

- Je dirais que oui. Les choses qui ne me plaisent pas, je ne les essaie pas. Peut-être, si je les essayais, elles finiraient par me plaire. Par exemple, les tomates.

- (À l'beure du repas), le plus important, c'est que la nourriture te plaise. Quand on parle de manger sainement, c'est un sacrifice, ça suppose beaucoup d'effort. Ça ne nous plaît pas.

Ainsi, les pratiques de régime, quand on les met en œuvre, supposent parfois l'élimination des aliments périphériques (gâteaux) ou un changement dans la manière de préparer les choses. Mais jamais, l'introduction d'un régime radicalement différent de celui de la famille:

\footnotetext{
${ }^{26}$ Je ne soutiens pas que ce type de pratiques est exclusif des classes populaires. Ces pratiques organisent certainement aussi la relation au corps d'agents qui vivent en d'autres points de l'espace social et ce à partir de combinaisons spécifiques avec la classe d'âge (on remarquera qu'elles sont formulées ici dans un groupe de discussion constitué de jeunes filles).

27 Sur l'importance du travail dans le système des goûts et sa transformation dans les carrières anorexiques, voir Darmon (2003, pp. 150-152 et 254).
} 
- Des fois, je me suis mise au régime. Comme des filets de viande grillée, j'élimine le pain ou je mange du pain complet.

- (Tu te passes) de desserts (...)

- Oui, tu manges les choses grillées. Mais les légumes et les fruits, tu ne les gô̂tes même pas.

c) Principe de définition sexuelle des surenchères corporelles. L'objectif de se sculpter un corps attirant se limite aux périodes de forte exposition au marché sexuel et matrimonial. Cette définition explicite du corps comme attribut sexuel élimine entièrement l'aura des pratiques corporelles ascétiques. En même temps, ce réalisme désenchanté, dans lequel il faut peut-être voir une réminiscence du vieux principe populaire de «ne pas se raconter des histoires », permet d'aborder l'autocontrôle corporel de manière temporaire et non comme résultat d'un impératif catégorique féminin 28.

- Nous, les femmes, nous devons plus attirer l'attention des bommes (...)

- Quand tu sais que tu as déjà ton mec, à toute épreuve, alors tu ne fais pas tant attention (à l'attirance physique).

- Tu fais attention, tu ne vas pas devenir une grosse vache, laide; (moi) j'essaie d'être bien. Mais si tu te négliges plus, tu te laisses plus aller, ça t'est plus égal.

d) Principe de réduction pratique de l'espace des possibles, affectif et sexuel. Ce réalisme inclut un fatalisme pratique. Quand on stabilise son couple, on cesse d'être « coté » sur le marché sexuel : les mots du groupe d'élèves cités plus haut (« une fois que tu as un mec, tu oublies ton corps») constituent un bon exemple du fonctionnement de ce principe. Une fois qu'on entre dans la «maturité », l'individu commence à négocier avec lui-même et avec ses proches la fin des tensions corporelles. Cette négociation exige une certaine homogénéité dans le groupe des proches. Dans le cas contraire, les forces de l'inquiétude corporelle peuvent recommencer à bouleverser le processus (continu et jamais définitif) du deuil par rapport à l'obtention d'un corps attirant selon les canons dominants. La distension des marchés tendus relègue les individus dans le monde dominant pour ce qui concerne le corps, mais, en même temps, elle les libère des processus de pression dans lesquels la maladie peut s'insinuer. Cette jeune participante au groupe de discussion le disait clairement à sa manière: le malheur et la tension pour être mince vont souvent de pair :

\footnotetext{
28 Dans les classes moyennes et les classes dominantes, j'entends souvent la thèse selon laquelle «toutes les femmes sont obligées d'être minces" et ce dans deux acceptions. La première est une acception critique d'origine féministe. La deuxième est que cette thèse permet à ceux qui l'émettent de se réaffirmer selon la nécessité inéluctable du contrôle corporel ascétique. Cette espèce de mauvaise foi, au sens que Sartre donnait à cette expression (l'individu se dissimule ses choix et prétend obéir à des forces qui le transcendent et qu'il est impossible de contrôler ou de moduler), est présente dans presque tous les entretiens que j'ai réalisés avec des femmes qui ont eu des troubles alimentaires. Je considère ces thèses (la pression corporelle est universelle et tout le monde la pratique) comme un agent actif dans la production de carrières corporelles tendues.
} 
Mais moi, je crois que tu commences aussi à grossir parce que tu n'as pas tellement de sujets de préoccupation, parce que comme tu vois des amies qui ont un fiancé et bon!... Moi, je n'ai pas de fiancé, je ne sais pas; ce n'est pas parce que tu manges, mais à cause des sujets que tu as en tête et qui te préoccupent. C'est la même chose que si un fiancé, par exemple, est impliqué dans les drogues ou ce genre de choses, je crois que la fille, même si elle l'aime beaucoup, comme il y a ce problème, la famille lui dit qu'elle doit laisser tomber ce garçon parce que ... Moi je crois que cette fille ne grossit pas simplement! Ce sont aussi les sujets qui la préoccupent: si un petit ami est bien, alors si aucun sujet ne la préoccupe, peut-être que la fille commence à grossir.

\section{Bibliographie}

Boltanski L. (1974). Puericultura y moral de clase, Barcelona, Laia.

Boltanski L. (1971). Les usages sociaux du corps, Annales: Économies, sociétés, civilisations, 26 (1), pp. 205-223.

Bourdieu P. (2002). Vous avez dit «populaire»? in: Langage et pouvoir symbolique, Paris, Seuil, pp. 132-151.

Bourdieu P. (1997). La ilusión biográfica, in: Razones prácticas. Sobre la teoría de la acción, Barcelona, Anagrama, pp. 74-83.

Bourdieu P. (avec Loïc J.-D. Wacquant) (1992). Réponses. Pour une anthropologie réflexive, Paris, Seuil.

Bourdieu P. (1988). La Distinción. Criterio y bases sociales del gusto, Madrid, Taurus.

Castel R. (1980). El psicoanalismo. El orden psicoanalítico y el poder, México, Siglo XXI.

Darmon M. (2003). Devenir anorexique. Une approche sociologique, Paris, La Découverte.

Faucher L. (1999). L'histoire de la folie à l'âge de la construction sociale. Compte rendu de L'âme réerite de Ian Hacking, Cabiers d'épistémologie, 9910, bttp://www.philo. uquam.ca

Goffman E. (1968). Asiles. Études sur la condition sociale des malades mentaux, Paris, Les Éditions de Minuit.

Goffman E. (1975). Stigmate. Les usages sociaux des handicaps, Paris, Les Éditions de Minuit.

Gremillion H. (2003). Feeding Anorexia. Gender and Power at a Treatment Center, Durham, Duke University Press.

Hacking I. (2002). Les fous voyageurs, Paris, Les Empêcheurs de penser en rond. 
Hacking I. (2001). ¿La construcción social de qué?, Barcelona, Paidós.

Hacking I. (1998). L'âme réécrite. Étude sur la personnalité multiple et les sciences de la mémoire, Paris, Les Empêcheurs de penser en rond.

Hoggart R. (1970). La culture du paurre. Étude sur les conditions de vie des classes populaires anglaises, Paris, Les Éditions de Minuit.

Maître J. (2000). Anorexie religieuse, anorexie mentale, Paris, Cerf.

Martín Criado E., Moreno Pestaña J.L. (2005). Conflictos sobre lo sano. Un estudio sociológico de la alimentación en las clases populares en Andalucía, Sevilla, Junta de Andalucía.

Mc Caughey M. (1999). Fleshing out the discomforts of femininity: The parallel cases of female anorexia and male compulsive bodybuilding, in: Weighty Issues. Fatness and Thinness as Social Problems, Sobal J., Maurer D. (eds), New York, Aldine de Gruyter, pp. 133-155.

Moreno Pestaña J.L. (2005). Jerarquías corporales, discursos científicos y construcción de identidad en los trastornos alimentarios. Un acercamiento sociológico, in: Nadie sabe lo que puede un cuerpo. Variaciones en torno al cuerpo y sus destinos, Corral N. (coord.), Madrid, Talasa, pp. 121-145.

Moulin R., Veyne P. (1996). Entretien avec Jean-Claude Passeron. Un itinéraire de sociologue, Revue Européenne des Sciences Sociales, Tome XXXIV, 103, pp. 275-354.

Parsons T. (1999). El sistema social, Madrid, Alianza Editorial.

Passeron J.-C. (1991). Le raisonnement sociologique. L'espace non-poppérien du raisonnement naturel, Paris, Nathan.

Passeron J.-C. (1970). Présentation, in : La culture du pauvre. Étude sur les conditions de vie des classes populaires anglaises, Hoggart R., Paris, Les Éditions de Minuit, pp. 7-26.

Remaury B. (2000). Le beau sexe faible. Les images du corps entre cosmétique et santé, Paris, Grasset.

Sánchez Moreno E. (2002). Sociología y enfermedad mental. Reflexiones en torno a un desencuentro, Revista Internacional de Sociología, 31, enero-abril, pp. 35-58.

Saukko P. (1999). Fat boys and goody girls: Hilde Bruch's work on eating disorders and the American anxiety about democracy, 1930-1960, in: Weighty Issues. Fatness and Thinness as Social Problems, Sobal J., Maurer D. (eds.), New York, Aldine de Gruyter, pp. 31-45.

Schwartz O. (2002). Le monde privé des ouvriers. Hommes et femmes du Nord, Paris, PUF (1re édition, 1990). 